\title{
The role of long chain fatty acids in regulating food intake and cholecystokinin release in humans
}

\author{
D Matzinger, L Degen, J Drewe, J Meuli, R Duebendorfer, N Ruckstuhl, M D’Amato, \\ L Rovati, C Beglinger
}

\begin{abstract}
Background and aims-The mechanism of intraduodenal fat induced inhibition of food intake is still unclear. Therefore, we tested the ability of duodenal fatty acids to suppress food intake at a lunchtime meal; in addition, we were interested to test if these effects were mediated by cholecystokinin (CCK) A receptors.

Subjects and methods-Three sequential double blind, three period crossover studies were performed in 12 healthy males each: (1) subjects received intraduodenal fat with or without $120 \mathrm{mg}$ of tetrahydrolipstatin, an inhibitor of gastrointestinal lipases, or saline; (2) volunteers received intraduodenal long chain fatty acids, medium chain fatty acids, or saline; (3) subjects received long chain fatty acids or saline together with concomitant intravenous infusions of saline or loxiglumide, a specific CCK-A receptor antagonist. The effect of these treatments on food intake and feelings of hunger was quantified.

Results-Intraduodenal fat perfusion significantly $(p<0.05)$ reduced calorie intake. Inhibition of fat hydrolysis abolished this effect. Only long chain fatty acids significantly $(p<0.05)$ decreased calorie intake, whereas medium chain fatty acids were ineffective. Infusion of loxiglumide abolished the effect of long chain fatty acids. Conclusions-Generation of long chain fatty acids through hydrolysis of fat is a critical step for fat induced inhibition of food intake; the signal is mediated via CCK-A receptors.

(Gut 2000;46:688-693)
\end{abstract}

Keywords: food intake; long chain fatty acids; medium chain fatty acids; cholecystokinin

The importance of fat storage and metabolism and its influences on long term control of energy balance in humans is well recognised. Welch and colleagues, ${ }^{1}$ in 1985 , observed in humans that infusion of a lipid emulsion into the ileum reduced food intake. They suggested that fat in the small intestine acts as a signal for short term control of food intake; an intravenous infusion of a similar lipid emulsion had no effect on eating. Further experiments with lipid infusions into the jejunum or ileum caused a decrease in food consumption, early satiation, ${ }^{23}$ and a delay in gastric emptying ${ }^{45}$; the effects were accompanied by an increase in plasma cholecystokinin (CCK) levels. ${ }^{3}$ These findings led to the hypothesis that fat acts at a preabsorptive site to decrease food intake and that this effect is indirectly mediated by release of CCK.

In parallel with these results, several animal experiments have been carried out. ${ }^{6-8}$ The effect of intraduodenal fat on food intake was extensively investigated in rats and pigs; more importantly, the effect was also studied in sham fed rats with an open gastric fistula. In this last experiment, the effect of intraduodenal fat on food intake was investigated independent of its effect on gastric emptying. The results of these experiments support the hypothesis of an intestinal signal induced by intraduodenal fat which initiates a reduction in food intake.

The major products of luminal lipid digestion are monoglycerides and fatty acids. ${ }^{9}{ }^{10}$ In humans, there is evidence that only fatty acids with chain lengths greater than $\mathrm{C} 10$ are effective in releasing CCK. ${ }^{11-13}$ Thus there is a link between fat digestion and the ability of fats to initiate a feedback response on food intake and release of CCK.

Hence the present study was designed to further understand the role of digestion products of intraduodenal fat in regulating food intake in humans. Three consecutive experimental series were performed. In the first series we tested the hypothesis that fat hydrolysis is a crucial step in the ability of lipid to initiate feedback inhibition of food intake. Tetrahydrolipstatin (THL) (also named Orlistat), a potent and irreversible inhibitor of gastrointestinal lipases, was used as a tool to prevent fat hydrolysis. ${ }^{14-16}$

In the second series we determined if the chain length of free fatty acids was crucial for initiating feedback inhibition of food intake. We used long chain fatty acids in the form of sodium oleate and medium chain fatty acids in the form of sodium caprylate to assess the importance of chain length.

In the third experimental series we tested the hypothesis that the feedback response on food intake induced by long chain fatty acids is mediated by CCK by using the specific CCK-A receptor antagonist loxiglumide (LOX).

\section{Materials and methods}

SUBJECTS

Thirty six male subjects, aged 20-40 years (mean 25.2), participated in the study. Body weight of all subjects was within the normal

Abbreviations used in this paper: ID, intraduodenal; LCF, long chain fatty acids; LOX loxiglumide; MCF, medium chain fatty acids; THL, tetrahydrolipstatin; CCK, cholecystokinin. 
range for age, sex, and height. Each subject gave written informed consent for the study. The protocol was approved by the human ethics committee of the University Hospital, Basel. Before acceptance, each participant was required to complete a medical interview, received a full physical examination, and participated in an initial laboratory screening. No subject was receiving any medications or had a history of food allergies or dietary restrictions.

EXPERIMENTAL PROCEDURE

Part I: effect of THL dissolved in olive oil on food intake

Three treatments, separated by at least seven days, were performed in each subject in a randomised order. On the evening preceding each experiment, subjects swallowed a radio-opaque polyvinyl feeding tube (external diameter 8 French gauge) which had an opening at the tip of the tube. The tube was inserted through the nose because this procedure allowed the tube to be retained overnight and for the duration of the experiment, but also allowed subjects to eat and drink with minimum discomfort. The tube was transported to the duodenum overnight. In the morning, the position of the tube was located fluoroscopically and the tip of the tube was positioned $100 \mathrm{~cm}$ distal to the teeth. It was firmly attached to the skin behind the ear to prevent further progression of the tube during the experiment. On the day of the experiment, each subject ate breakfast if this was his normal custom but no snacks were allowed after 8 am. At noon, the experiment started with continuous perfusion to the duodenum. The treatments were identical in design except for the intraduodenal perfusions.

One treatment consisted of intraduodenal saline perfusion for the duration of the experiment. Sixty minutes after starting the perfusion, subjects were invited to eat and drink as much as they wished. In the second and third experiments, intraduodenal fat (olive oil) with or without $120 \mathrm{mg}$ THL was used instead of saline throughout the experiments. A perfusion rate of $0.5 \mathrm{ml} / \mathrm{min}$ (load $41 \mathrm{~g}$ ) was chosen for a total of 90 minutes; this rate was taken from previous experiments. ${ }^{4}{ }^{6}$ The meal consisted of: (a) orange juice; (b) ham sandwiches (60 g wheat bread, $10 \mathrm{~g}$ butter, and $25 \mathrm{~g}$ ham); (c) chocolate pudding; and (d) coffee with cream and sugar (coffee could be sweetened if desired; therefore cream and sugar were optional). The order of food intake had to follow the above schedule. To reduce the participant's awareness of the amount of food eaten, food was presented in small samples and in excess. The intraduodenal fat perfusion solution was indistinguishable in appearance from the control solution (saline). The investigator was unaware of the respective treatments thereby making it possible to perform treatments in a double blind manner. The amount of food eaten, volume of fluid drunk, and time for each subject to complete the meal were quantified. From these observations the total calorie intake was calculated.
Subjects scored their subjective feelings of hunger and fullness at 15 minute intervals for the duration of each experiment using a visual analogue scale from 1 to 10 and indicated their scores on a questionnaire. The scale and scores have previously been designed and described in detail. $^{13}$

Part II: effect of free fatty acids on food intake The design of the second series was similar to part I. The experiment was a randomised, double blind, three period crossover study in 12 healthy male subjects. One treatment consisted of intraduodenal perfusion of free medium chain fatty acids (MCF). MCF in the form of sodium caprylate, a fatty acid with eight carbons, was perfused at a concentration of $0.049 \mathrm{~g} / \mathrm{ml}$ at a rate of $0.5 \mathrm{ml} / \mathrm{min}$, resulting in a load of $8 \mathrm{mmol} / \mathrm{h}$ sodium caprylate; this load is equivalent to $26 \mathrm{kcal} / \mathrm{h}$. In the second experiment, intraduodenal perfusion of free long chain fatty acids (LCF) was used. LCF in the form of sodium oleate, a long chain fatty acid with 18 carbons, was perfused at a concentration of $0.086 \mathrm{~g} / \mathrm{ml}$ at a rate of $0.5 \mathrm{ml} / \mathrm{min}$, resulting in a load of $8 \mathrm{mmol} / \mathrm{h}$; this load is equivalent to $46 \mathrm{kcal} / \mathrm{h}$. On the third experimental day, volunteers received intraduodenal saline (control) instead of free fatty acids. Sixty minutes after starting the respective perfusions, subjects were invited to eat and drink as much as they wished. The MCF and LCF loads were chosen from previous experiments. ${ }^{17}$ These data have shown that an intraduodenal rate of $8 \mathrm{mmol} / \mathrm{h}$ sodium oleate induces maximal gall bladder contraction in healthy human subjects. During the experiments, $7.5 \mathrm{ml}$ of blood were drawn into ethylendiaminetetra-acetic acid (EDTA) coated tubes containing aprotinin (1000 KIU/ml blood) for pre-meal plasma CCK and leptin determinations (fasting blood sample and 25, 15, and 5 minutes before the start of the meal).

\section{Part III: effect of LCF with and without intravenous LOX on food intake}

The procedures in this series were similar to part II except for the intravenous infusions and intraduodenal perfusions (MCF were not perfused in this part). Twelve healthy male subjects participated in this randomised double blind study. Subjects received on two experimental days continuous intraduodenal perfusion of LCF ( $8 \mathrm{mmol} / \mathrm{h}$ sodium oleate for a total of 90 minutes) together with either an intravenous infusion of isotonic saline (control) or an infusion of the CCK-A receptor antagonist LOX $(10 \mathrm{mg} / \mathrm{kg} / \mathrm{h})$ for the duration of the experiment. The dose of LOX was chosen from previous experiments. ${ }^{3}{ }^{18} 19$ Infusions were started 30 minutes before intraduodenal perfusion. On the third experimental day, subjects received intraduodenal saline and intravenous saline during the experiment. After 60 minutes of perfusion, volunteers were allowed to eat and drink as much as they liked. Infusions were delivered by ambulatory infusion pumps through a Teflon catheter inserted into a forearm vein. Blood was taken $(7.5 \mathrm{ml})$ at regular intervals for pre-meal hormone determinations 
(fasting blood sample and 25, 15, and 5 minutes before the start of the meal).

Plasma hormone determinations

Plasma CCK concentrations were measured using a sensitive radioimmunoassay based on the antiserum OAL656 which recognises the sulphated tyrosine residue of CCK8 but has no relevant cross reactivity with sulphated gastrin $(<1 \%)$ and does not cross react with unrelated gastrointestinal peptides. ${ }^{20}$ Plasma samples were extracted with ethanol. The detection limit of the assay was $3 \mathrm{pmol} / \mathrm{ml}$ plasma using CCK33 as a standard. Details of the assay have been described previously. ${ }^{18}$ Plasma leptin levels were assayed using a commercial kit (Linco Ltd, St Louis, Missouri, USA).

STATISTICAL ANALYSIS

The amount of food eaten and amount of fluid drunk, including their corresponding energy intake ( $\mathrm{kcal})$, were compared between treatments by analysis of variance (ANOVA). For significant differences, multiple paired $t$ tests with Bonferroni's correction were performed. The same statistical procedure was used to analyse the results of plasma hormone concentrations using area under the curve analysis and scores for hunger and fullness. ${ }^{21}$

\section{Results}

FOOD INTAKE

Part I

The amount of food eaten (20\% reduction) and the corresponding calorie intake $(23 \%$ reduction) were significantly $(\mathrm{p}<0.01)$ reduced after intraduodenal (ID) fat administration compared with the control experiment (ID saline) (table 1). THL (120 mg perfused with fat) reversed this effect and caused similar food and calorie intake as the ID saline treatment. These data indicate that the reduction in

Table 1 Effect of intraduodenal (ID) fat, with or without $120 \mathrm{mg}$ THL, or saline on eating behaviour in 12 healthy male subjects

\begin{tabular}{lrrc}
\hline & ID Saline & ID Fat & ID Fat plus THL \\
\hline Calorie intake (kcal) & $1422(82)$ & $1097(88)^{\star}$ & $1345(69) \dagger$ \\
Amount of food ingested (g) & $433(32)$ & $347(32)^{\star}$ & $430(29)$ \\
Amount of fluid intake (ml) & $633(74)$ & $658(63)$ & $634(63)$ \\
\hline Data are mean (SEM). & & & \\
${ }^{*} \mathrm{p} \leqslant 0.05 v$ control (ID saline); $\dagger \mathrm{p} \leqslant 0.05 v$ ID fat. & &
\end{tabular}

Table 2 Effect of intraduodenal (ID) MCF, LCF, or saline on eating behaviour in 12 healthy male subjects

\begin{tabular}{lccc}
\hline & ID Saline & ID MCF & ID LCF \\
\hline Calorie intake (kcal) & $2191(100)$ & $2120(140)$ & $1813(105)^{\star}$ \\
Amount of food ingested (g) & $753(45)$ & $719(70)$ & $609(49)$ \\
Amount of fluid intake (ml) & $733(41)$ & $763(72)$ & $668(58)$ \\
\hline
\end{tabular}

Data are mean (SEM).

${ }^{\star} \mathrm{p} \leqslant 0.05 v$ control (ID saline).

Table 3 Effect of intraduodenal (ID) LCF or saline with an intravenous (IV) infusion of saline or LOX on eating behaviour in 12 healthy male subjects

\begin{tabular}{lccc}
\hline & $\begin{array}{l}\text { ID Saline plus IV } \\
\text { saline }\end{array}$ & $\begin{array}{l}\text { ID LCF plus IV } \\
\text { saline }\end{array}$ & $\begin{array}{l}\text { ID LCF plus IV } \\
\text { LOX }\end{array}$ \\
\hline Calorie intake (kcal) & $2056(132)$ & $1796(144)^{\star}$ & $2075(162) \dagger$ \\
Amount of food ingested (g) & $666(55)$ & $568(47)$ & $720(62) \dagger$ \\
Amount of fluid intake $(\mathrm{ml})$ & $890(59)$ & $828(60)$ & $919(69)$ \\
\hline
\end{tabular}

Data are mean (SEM)

${ }^{\star} \mathrm{p} \leqslant 0.05 v$ control (ID saline plus IV saline); $\mathrm{t} \mathrm{p} \leqslant 0.05 v$ ID LCF.

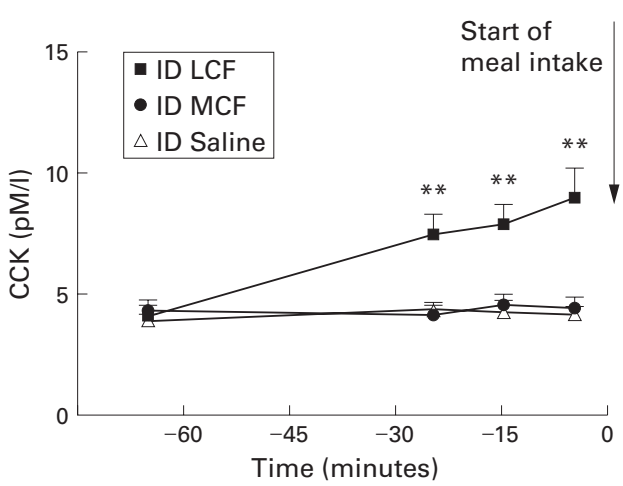

Figure 1 Plasma cholecystokinin (CCK) concentrations in the pre-meal period during intraduodenal (ID) perfusions of MCF, LCF, or saline in 12 healthy male subjects. Data are mean (SEM). **Significant increase in plasma CCK levels during LCF perfusion compared with saline $(p<0.01)$.

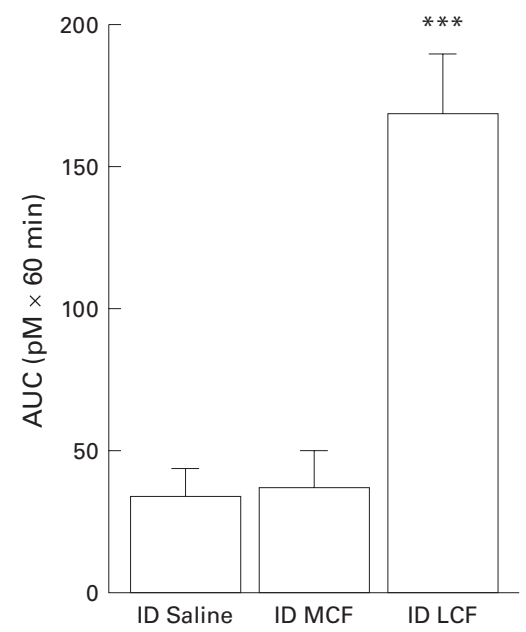

Figure 2 AUC of plasma cholecystokinin (CCK) responses to intraduodenal (ID) perfusion of saline, $M C F$, or LCF during the pre-meal period in 12 healthy male subjects. Results are mean (SEM). ${ }^{* *}$ Significant difference between ID LCF $v$ ID saline administration (AUC: $p \leqslant 0.0001$ ).

energy consumption induced by ID fat administration can be abolished by inhibition of fat hydrolysis. Fluid intake and meal duration were not influenced (data not shown).

Part II

Perfusion of ID LCF resulted in a reduction in food $(19 \%)$ and calorie $(17 \%)$ intake compared with the control experiment (ID saline); however, the effect was significant $(p<0.05)$ only for calorie consumption and not for the amount of food eaten (table 2) due to variability $(p=0.058)$. Fluid intake was not changed with ID LCF. In contrast with LCF perfusion, ID MCF did not affect food or fluid consumption or calorie intake compared with the control experiment. Meal duration was not influenced by any treatment (data not shown).

Part III

A significant $(\mathrm{p}<0.05)$ reduction in calorie intake was seen after ID LCF with IV saline administration compared with the control experiment (ID saline with IV saline), similar to the results in part II (tables 2, 3). IV LOX reversed the inhibition induced by LCF and 


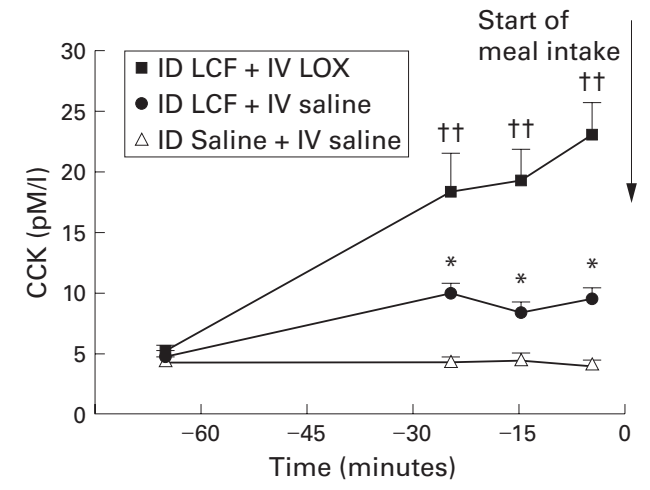

Figure 3 Plasma cholecystokinin (CCK) concentrations in the pre-meal period during intraduodenal (ID) perfusion of $L C F$ with intravenous (IV) saline, ID LCF with IV LOX, or ID saline with IV saline in 12 healthy male subjects. Data are mean (SEM). * Significant increase in plasma CCK between ID LCF + IV saline v ID saline + IV saline $(p<0.05)$; + tsignificant increase in plasma CCK levels between ID saline $+I V$ saline v ID LCF + IV LOX $(p<0.01)$.

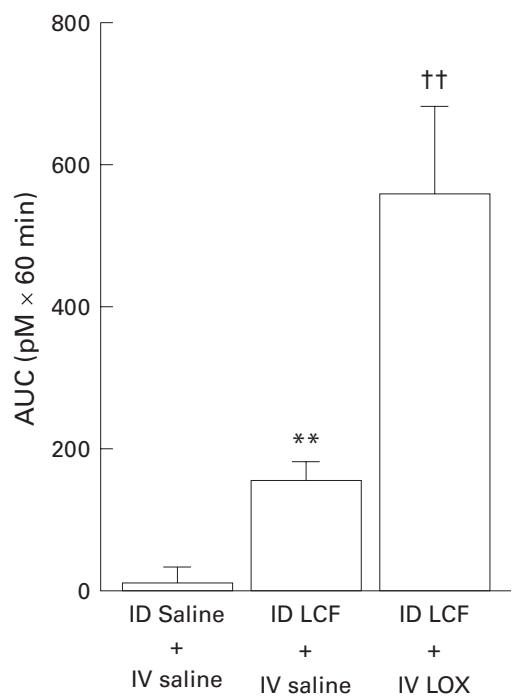

Figure 4 AUC of plasma cholecystokinin (CCK) responses to intraduodenal (ID) perfusion of saline with intravenous (IV) infusion of saline, ID LCF with IV saline, or ID LCF with IV LOX during the pre-meal period in 12 healthy male subjects . Results are mean

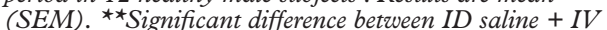
saline v ID $L C F+I V$ saline $(A U C: p<0.01)$;

t+significant difference between ID saline $+I V$ saline $v$ ID $L C F+I V L O X(A U C: p<0.005)$.

caused similar calorie and food consumption as in the control experiment; however, the results were significantly different $(\mathrm{p}<0.05)$ from administration of ID fat with IV saline (table 3). These data indicate that the reduction in calorie and food intake induced by ID fat administration can be blocked by the CCK-A receptor antagonist LOX.

PLASMA HORMONES

Part II

During the control experiment and ID perfusion of MCF, plasma CCK levels remained stable in the pre-meal period (fig 1). Administration of ID LCF caused a significant increase in plasma CCK levels compared with controls (AUC: $\mathrm{p} \leqslant 0.0001$ ) (fig 2).
Fasting plasma leptin concentrations were similar in the experiments and did not change with the different treatments (data not shown).

Part III

In the experiment with ID LCF and IV LOX, plasma CCK secretion during the pre-meal period resulted in a significant and augmented increase (fig 3) in comparison with ID saline with IV saline (AUC: $p \leqslant 0.001$ ). Similar to part II, ID perfusion of LCF with IV infusion of saline caused a significant increase in plasma CCK (AUC: $p \leqslant 0.001$ ) compared with the control experiment (fig 4).

Leptin concentrations were similar and did not change during the three treatments (data not shown).

\section{EATING BEHAVIOUR}

In all three experimental series (parts I, II, and III) fat or LCF perfusion induced feelings of early satiety compared with saline perfusion, but the effects were not significant; in fact, no significant effects on hunger and fullness ratings were observed between the different treatments (data not shown).

\section{Discussion}

Satiety can be influenced by intraduodenal fat administration. However, in humans it is not yet clear if the satiety effect of fat is produced by intact triglycerides or its digestive products. The influence of intraduodenally perfused triolein, oleic acids with monoolein (digestion products of triolein), and oleic acid on meal patterns has been investigated in ad libitum fed rats. ${ }^{22}$ The results revealed that the effects of oleic acids with monoolein on reduction in meal size and meal frequency were similar to those produced by oleic acid alone, but fourfold more potent than triolein delivery to the small intestine. These results support the hypothesis that the satiation response to duodenal triolein is produced by the products of triolein digestion. In contrast with the above results, intraduodenal infusion of $20 \%$ Intralipid (an emulsion containing primarily long chain triglycerides) and monoglyceride inhibited food intake more effectively than oleic acid in pigs. ${ }^{8}$ It is not clear if these contradictory data reflect species differences or differences in experimental design. Data from these animal studies formed the basis of our investigation.

The crucial importance of fat digestion products on digestive function is best illustrated by their effects on exocrine pancreatic secretory responses in rats, ${ }^{23}{ }^{24} \mathrm{dogs},{ }^{25}$ and humans ${ }^{26}$ in which duodenal infusion of long chain free fatty acids alone can stimulate maximal pancreatic enzyme and bicarbonate secretion $^{23}{ }^{24}$ whereas undigested long chain triglycerides are ineffective. ${ }^{26}$ Furthermore, inhibition of lipolysis reduces the amount of free fatty acids in the small intestine with a subsequent reduction in CCK release. ${ }^{26}$ The reduction in CCK release inhibits gall bladder contraction and lowers postprandial pancreatic exocrine secretory responses. Spannagel and colleagues $^{24}$ showed that intraduodenally perfused fat is not only a potent stimulant of 
pancreatic exocrine secretory responses and plasma CCK secretion in fat adapted rats, but that the enhanced response to fat in these animals was mediated by increased secretion of lipolytic enzymes, which in turn could increase the rate of triglycerides to fatty acids in the small intestine. Taken together, these results imply that free fatty acids are crucial for stimulation of CCK release, gall bladder contraction, and pancreatic exocrine secretory responses.

In agreement with these observations, we have seen here that long chain fatty acids are also crucial for inducing effects on food intake. The experiments largely confirm the observations in rats that hydrolysis of fat is necessary for stimulation of fat induced satiation. ${ }^{22}$ Intraduodenal administration of fat caused a marked reduction in calorie intake and food consumption. THL, a tool which prevented lipolysis, abolished the effect of ID fat on food consumption. Hildebrand et al found that perfusion of triglycerides concomitant with THL resulted in marked suppression of CCK secretion. ${ }^{26}$ As CCK has been shown to induce effects on satiety, we believe that the effects initiated by ID fat are mediated by this peptide. Definite evidence, based largely on studies with exogenous CCK, support the following: (1) CCK reduces meal size in animals ${ }^{27-32}$ and humans ${ }^{19}{ }^{33}$; and (2) type A receptors seem to be critical. ${ }^{1934} 35$ From these results we conclude that the process of satiation is dependent on fat digestion with concomitant release of CCK.

To further support our conclusions, a second approach was taken to investigate the role of free fatty acids on satiety in humans. In this part of the study MCF or LCF were perfused into the small intestine. LCF perfusion resulted in a reduction in calorie intake and food consumption concomitant with a significant increase in plasma CCK concentrations. $\mathrm{MCF}$ perfusion was ineffective: there was no effect on food intake or plasma CCK release. These observations suggest that in addition to fat hydrolysis, the chain length of free fatty acids is crucial for initiating an effect on satiety.

The availability of LOX, a potent and selective CCK-A receptor antagonist, has made it possible to test the last part of the hypothesis that CCK is mediating the effects of intraduodenal LCF via peripheral CCK-A receptors. As expected, ID LCF significantly reduced food consumption and calorie intake. Concomitant application of LOX completely abolished the satiation effect of LCF and caused a similar calorie intake as in the control experiment. In rats, the inhibitory effect of intraduodenal sodium oleate ${ }^{7}$ or Intralipid ${ }^{34}$ on food intake was antagonised by CCK-A receptor antagonists. Similarly, LOX largely prevented the inhibitory effect of intraduodenal Intralipid on food intake in humans. ${ }^{19}$ Our present data therefore support and extend these findings: LCF initialises the satiation signal to the brain through release of endogenous CCK; CCK then acts on CCK-A receptors which have been identified on the abdominal vagus. ${ }^{36}$ It is conceivable that direct activation of afferent fibres through LCF could be an alternative pathway. One type of afferent fibres is sensitive to long chain fatty $\operatorname{acids}^{37}$; furthermore, inhibition of food intake induced by intraduodenal sodium oleate is reversed by bilateral, subdiaphragmatic vagotomy ${ }^{38}$ or pretreatment with capsaicin. ${ }^{35}$ It is not clear, however, how CCK could be involved in this last scheme.

Recent evidence has suggested that leptin, the product of the obese gene, may be involved in satiety pathways originating from the gastrointestinal tract. Bado and colleagues ${ }^{39}$ detected leptin gene expression and immunoreactivity in the gastric fundus. Furthermore, food ingestion caused rapid stimulation of gastric leptin secretion, an effect which was reproduced by CCK administration. In mice, leptin enhances the satiety inducing effect of $\mathrm{CCK}^{40}$ suggesting that CCK induced leptin secretion may amplify the intestinal regulation of food intake. In the present study, however, we did not observe any changes in circulating leptin concentrations.

Previous animal studies have suggested that the satiation effect of ID fat occurs before digestion products of fat enter into the bloodstream; based on this observation it was concluded that the effects of ID fat are independent of fat metabolism. ${ }^{41}{ }^{42}$ Lipids are predominantly absorbed into the lymphatics and they then enter into the circulation through the superior vena cava (carbon chain length of $14 \mathrm{C}$ or more) or are absorbed directly into the hepatic portal vein (carbon chain length shorter than $14 \mathrm{C}$ ). ${ }^{43-45}$ In our study, neither MCF nor LCF affected concentrations of free fatty acids in plasma compared with the control experiments (data not shown). Based on these results, the hypothesis of a preabsorptive role for fat induced satiation in humans is supported.

In conclusion, the results of our studies emphasise the importance of fat digestion in the regulation of fat induced satiation in humans. Furthermore, we found that only long chain free fatty acids can initialise this process, with CCK acting through its A receptor to mediate this effect.

We thank Silvia Ketterer and Gerdien Gamboni for expert technical assistance and Carita Frei for excellent editorial assistance. This work was supported by a grant from the Swiss National Science Foundation (grant No 3200-40604-94/1). This study was presented in part at the Annual Meeting of the American was presented in part at the Annual Meeting of the American Gastroenterological Assoc
lished in abstract form.

1 Welch I, Saunders $\mathrm{K}$, Read NW. Effect of ileal and intravenous infusion of fat emulsion on feeding and satiety

in human volunteers. Gastroenterology 1985;89:1293-7.
Welch I, Seeple CP, Read NW. Comparison of the effect on Welch I, Seeple CP, Read NW. Comparison of the effect on
satiety and eating behaviour of infusion of lipid into the different regions of the small intestine. Gut 1988;29:306-11

3 Drewe J, Gadient A, Rovati LC, Beglinger C. Role of circulating cholecystokinin in control of fat-induced inhibition of food intake in humans. Gastroenterology 1992;102:16549.

4 Read NW, McFarlane A, Kinsman RI, Bloom SR. Effect of infusion of nutrient solutions into the ileum on gastrointestinal transit and plasma levels of neurotensin and enteroglucagon. Gastroenterology 1984;86:274-80.

5 Miller LJ, Malagelada JR, Taylor WF, Go VWL. Intestinal control of human postprandial gastric function. The role of components of jejuno-ileal chyme in regulating gastric and gastric emptying. Gastroenterology $1981 ; 80$ : ecretion and gastric emptying. Gastroenterology 1981;80: $763-9$

6 Greenberg D, Smith GP, Gibbs J. Intraduodenal infusion of fats elicts satiety in the sham-feeding rat. Am $\mathcal{F}$ Physiol 1990;259:R110-18.

7 Yox DP, Stokesberry HRCR. Supression of sham feeding by intraintestinal oleat: blockade by a CCK antagonist and 
reversal of blockade by exogenous CCK-8. In: Hughes J, Dockray G, Woodruff G, eds. The neuropeptide cholecystokinin (CCK), anatomy and biochemistry, receptors, pharmacology and physiology. Chinchester: Horwood, 1989:218-22.

8 Gregory PC, Rayner DV. The influence of gastrointerstinal infusion of fats on regulation of food intake in pigs. F Physiol 1987;385:471-8

9 Staggers JE, Hernell O, Stafford RJ, Carey MC. Physicalchemical behavior of dietary and biliary lipids during intestinal digestion and absorption: 1. Phase behavior and aggregation states of model lipid systems patterned after aqueous duodenal contents of healthy adults. Biochemistry 1990;29:2028-40.

10 Hernell O, Staggers JE, Carey MC. Physical-chemical behavior of dietary and biliary lipids during intestinal digestion and absorption: 2. Phase analysis and aggregation digestion and absorption: healthy adult human beings. Biochemistry 1990;29:204156.

11 Hopman WP, Jansen JBMJ, Rosenbusch G, Lamers CBHW Effect of equimolar amounts of long-chain triglycerides and medium-chain triglycerides on plasma cholecystokinin and medium-chain triglycerides on plasma cholecystokinin

12 Isaacs PE, Ladas S, Forgacs IC Comparison of effects of ingested medium- and long-chain triglyceride on gallbladder volume and release of cholecystokinin and other gut peptides. Dig Dis Sci 1987;32:481-6.

13 McLaughin J, Grazia Luca MM, Jones MN, D'Amato M, Dockray GJ, Thompson DG. Fatty acid chain length determines cholecystokinin secretion and effect on human gastric motility. Gastroenterology 1999;116:46-53.

14 Borgström B. Mode of action of tetrahydrolipstatin: a derivative of the naturally occurring lipase inhibitor lipstatin. Biochim Biophys Acta 1988;962:308-16.

15 Hadváry $\mathrm{P}$, Lengsfeld $\mathrm{H}$, Wolfer $\mathrm{H}$. Inhibition of pancreatic lipase in vitro by the covalent inhibitor tetrahydrolypstatin. Biochem 7 1988;256:357-61.

16 Güzelhan C, Crijns HJMJ, Peters PAM, Jonkman JHG, Hartmann D. Pharmacological activity (inhibition of fat Hartmann D. Pharmacological activity (inhibition of fat absorbtion) and tolerability in healthy volunteers of tetrahydrolipstatin (THL)-a specific lipase
stract). Int 尹 Obesity 1991;15 (suppl 1):29.

17 Otth S. Inauguraldissertation: Hormonale versus cholinerge Mechanismen in der Regulation der fettstimulierten Pankreassekretion und der Gallenblasenkontraktion beim Menschen. Basel: Medizinische Fakulät der Universität Basel, 1995.

18 Meier R, Hildebrand P, Thumshirn M, et al. Effect of loxiglumide, a cholecystokinin antagonist, on pancreatic polypeptide release in humans. Gastroenterology 1990;99: 1757-62.

19 Lieverse RJ, Jansen JB, Masclee AA, Rovati LC, Lamers CB. Effect of a low dose of intraduodenal fat on satiety in humans: studies using the type A cholecystokinin receptor antagonist loxiglumide. Gut 1994;35:501-5.

20 Hashimura E, Shimizu F, Nishino T, Imagawa K, Tateishi K, Hamaoka T. Production of rabbit antibody specific for aminoterminal residues of cholecystokinin octapeptide (CCK-8) by selective suppression of cross-reactive antibody response. F Immunol Methods 1982;55:375-87.

21 body response. F Imminol Statistik. Berlin: Springer, 1984.

21 Sachs L. Angewandte Statistik. Berlin: Springer, 1984 . cholecystokinin in the anorexia produced by duodenal cholecystokinin in the anorexia produced by duodenal
delivery of oleic acid in rats. Am $\mathcal{F}$ Physiol $1995 ; 269(6 \mathrm{Pt} 2)$ delivery of

23 Demol P, Sarles H. Action of fatty acids on the exocrine pancreatic secretion of the conscious rats: further evidence for a protein pancretic inhibitory factor. 7 Physiol Lond 1978;275:27-37.
24 Spannagel AW, Nakano I, Tawil T, Chey WJ, Liddle RA, Green GM. Adaptation to fat markedly increases pancretic secretory response to intraduodenal fat in rats. $A m \mathcal{F}$ atic secretory response to intr.
Physiol 1996;270:G128-35.

25 Meyer JH, Jones RS. Canine pancreatic responses to intestinally perfused fat and products of fat digestion. $A m \mathcal{F}$ Physiol 1974;226:1178-87.

26 Hildebrand P, Petrig C, Burckhardt B, et al. Hydrolysis of dietary fat by pancreatic lipase stimulates cholecystokinin release. Gastroenterology 1998;114:123-9.

27 Gibbs J, Young RC, Smith GP. Cholecystokinin elicits satiety in rats with open gastric fistulas. Nature 1973;245:3235.

28 Gibbs J, Falasco JD, McHugh PR. Choleystokinin decreases food intake in rhesus monkeys. Am $\mathcal{F}$ Physiol 1976;230:1518.

29 Weatherford SC, Chiruzzo FY, Laughton WB. Satiety induced by endogenous and exogenous cholecystokinin is mediated by CCK-A receptors in mice. Am f Physiol 1992; 262:R574-8.

30 Moran TH, Ameglio PJ, Schwartz GJ, McHugh PR. Blockade of type A, not type B, CCK receptors attenuates the satiety actions of exogenous and endogenous CCK. Am 7 satiety actions of exogenous
Physiol 1992;262:R46-50.

31 Foltin RW, Moran TH. Food intake in baboons: effect of a long-acting cholecystokinin analog. Appetite 1989;12:14552

32 Figlewicz DP, Sipols AJ, Porte D, Woods SCJ, Liddle RA. ntraventricular CCK inhibits food intake and gastric emptying in the baboon. Am f Physiol 1989;256:R1313-17.

33 Kissileff HR, Pi-Sunyer FX, Thornton J, Smith GP. C-terminal octapeptide of cholecystokinin decreases food intake in man. Am 7 Clin Nutr 1981;34:154-60.

34 Greenberg D, Torres NJ, Smith GP, Gibbs J. The satiating effects of fats is attenuated by the cholecystokinin antagonist lorglumide. Ann NY Acad Sci 1989;575:517-20. 5 Yox DP, Stockesberry H, Ritter RC. Fourth ventricular cap-
saicin attenuates suppression of sham feeding induced by intestinal nutrients. Am F Physiol 1991;260:R681-7.

36 Moran TH, Smith GP, Hostetler AM. Transport of cholecystokinin (CCK) binding sites in subdiaphragmatic vagal branches. Brain Res 1987;415:149-52.

37 Melone J. Vagal receptors sensitive to lipids in the small intestine of the cat. F Auton Nerv Syst 1986;17:331-41.

38 Yox DP, Stockesberry H, Ritter RC. Vagotomy attenuates suppression of sham feeding induced by intestinal nutrients. Am f Physiol 1991;260:R503-8.

39 Bado A, Levasseur S, Attoub S, et al. The stomach is a source of leptin. Nature 1998;394:790-3.

40 Barrachina MD, Martinez V, Wang L, Wei JT, Taché Y. Synergistic interaction between leptin and cholecystokinin to reduce short-term food intake in mice. Proc Natl Acad Sci USA 1997;94:10455-60.

41 Greenberg D, Kava RA, Lewis DR, Greenwood MRC, Smith GP. Time course for entry of intestinally infused lipids into blood of rats. Am F Physiol 1995;269:R432-6.

42 Greenberg D, Smith GP, Gibbs J. Intravenous triglycerides fail to elicit satiety in sham-feeding rats. Am F Physiol 1993; 264:R409-13.

43 Senior JR. Intestinal absorption of fats. $\mathcal{F}$ Lipid Res 1964;5:495-521.

44 Nicholl CG, Polak JM, Bloom SR. The hormonal regulation of food intake, digestion and absorption. Annu Rev Nutr 1985;5:213-39.

45 Clark SB, Brause B, Holt PR. Lipolysis and absorption of fat in the rat stomach. Gastroenterology 1969;56:214-22. 